\title{
Hypoxia promotes fibrogenesis in vivo via HIF-1 stimulation of epithelial-to-mesenchymal transition
}

\author{
Debra F. Higgins, ${ }^{1}$ Kuniko Kimura, ${ }^{2}$ Wanja M. Bernhardt, ${ }^{3}$ Nikita Shrimanker, ${ }^{1}$ Yasuhiro Akai, ${ }^{1,2}$ \\ Bernd Hohenstein, ${ }^{3}$ Yoshihiko Saito, ${ }^{2}$ Randall S. Johnson, ${ }^{4}$ Matthias Kretzler, ${ }^{5}$ Clemens D. Cohen, ${ }^{6}$ \\ Kai-Uwe Eckardt,, ${ }^{3}$ Masayuki Iwano, ${ }^{2}$ and Volker H. Haase ${ }^{1}$

\begin{abstract}
1Department of Medicine, Renal Electrolyte and Hypertension Division, University of Pennsylvania, Philadelphia, Pennsylvania, USA. 2Department of Internal Medicine, Nara Medical University, Kashihara, Nara, Japan. ${ }^{3}$ Department of Nephrology and Hypertension, Friedrich-Alexander University Erlangen-Nuremberg, Erlangen, Germany. ${ }^{4}$ Division of Biology, UCSD, La Jolla, California, USA. ${ }^{5}$ Department of Internal Medicine, Division of Nephrology, University of Michigan Medical School, Ann Arbor, Michigan, USA.
\end{abstract} \\ ${ }^{6}$ Nephrologisches Zentrum, Medizinische Poliklinik, University of Munich, Munich, Germany.
}

\begin{abstract}
Hypoxia has been proposed as an important microenvironmental factor in the development of tissue fibrosis; however, the underlying mechanisms are not well defined. To examine the role of hypoxia-inducible factor-1 (HIF-1), a key mediator of cellular adaptation to hypoxia, in the development of fibrosis in mice, we inactivated Hif- $1 \alpha$ in primary renal epithelial cells and in proximal tubules of kidneys subjected to unilateral ureteral obstruction (UUO) using Cre-loxP-mediated gene targeting. We found that Hif-1 $\alpha$ enhanced epithelial-tomesenchymal transition (EMT) in vitro and induced epithelial cell migration through upregulation of lysyl oxidase genes. Genetic ablation of epithelial Hif-1 $\alpha$ inhibited the development of tubulointerstitial fibrosis in UUO kidneys, which was associated with decreased interstitial collagen deposition, decreased inflammatory cell infiltration, and a reduction in the number of fibroblast-specific protein-1-expressing (FSP-1-expressing) interstitial cells. Furthermore, we demonstrate that increased renal HIF-1 $\alpha$ expression is associated with tubulointerstitial injury in patients with chronic kidney disease. Thus, we provide clinical and genetic evidence that activation of HIF-1 signaling in renal epithelial cells is associated with the development of chronic renal disease and may promote fibrogenesis by increasing expression of extracellular matrix-modifying factors and lysyl oxidase genes and by facilitating EMT.
\end{abstract}

\section{Introduction}

Peritubular capillary loss and reduced blood flow limit oxygen supply to the renal interstitium, leading to chronic interstitial and tubular cell hypoxia, which can initiate and sustain interstitial scarring and tubular atrophy. This is evident in renal biopsy samples from patients with chronic kidney disease (CKD), which typically display loss of peritubular capillaries in areas of tubulointerstitial fibrosis (1-3). Thus, renal hypoxia was considered a consequence rather than an inducer of the fibrogenic process. However, recent data suggest that hypoxia may represent an early and potentially initiating event in the development and progression of renal disease (4-6), although the underlying molecular mechanisms by which this occurs remained unclear.

A key mediator of cellular responses to low oxygen is hypoxiainducible factor-1 (HIF-1), a heterodimeric transcription factor consisting of a constitutively expressed $\beta$ subunit (also known as aryl hydrocarbon receptor nuclear translocator [ARNT]) and an $\mathrm{O}_{2}$-regulated $\alpha$ subunit. In the presence of oxygen, prolyl hydroxylase domain (PHD) enzymes hydroxylate HIF-1 $\alpha$ and enable inter-

Nonstandard abbreviations used: BAPN, $\beta$-aminopropionitrile; BCS, bathocuproine disulfonate; $\mathrm{CKD}$, chronic kidney disease; CTGF, connective tissue growth factor; DN, diabetic nephropathy; EMT, epithelial-to-mesenchymal transition; EPO, erythropoietin; FSP-1, fibroblast-specific protein-1; HIF-1, hypoxia-inducible factor-1; PTEC primary tubular epithelial cell; pVHL, von Hippel-Lindau protein; UUO, unilateral ureteral obstruction.

Conflict of interest: The authors have declared that no conflict of interest exists. Citation for this article: J. Clin. Invest. 117:3810-3820 (2007). doi:10.1172/JCI30487. action with von Hippel-Lindau protein (pVHL), which results in its ubiquitylation and subsequent proteasomal degradation. When oxygen is limited, hydroxylation of HIF- $1 \alpha$ is inhibited, thus preventing PVHL interaction, which results in stabilization and translocation of HIF- $1 \alpha$ into the nucleus, where it dimerizes with HIF-1 $\beta$ to form active HIF-1 transcription factor (reviewed in ref. 7). In addition to hypoxic stabilization of HIF- $1 \alpha$, several factors relevant to renal fibrogenesis, including nitric oxide, TNF- $\alpha(8,9)$, angiotensin II (10), IL-1 (11), insulin and insulin-like growth factors (11-13), have been shown to induce nonhypoxic HIF-1 $\alpha$ stabilization. Therefore, it is plausible that HIF-1 $\alpha$ stabilization can occur even in the absence of tissue hypoxia and lends further significance to the role that HIF-1 may play in disease progression in vivo.

HIF-1 and its homolog HIF-2, which can be found in renal interstitial, endothelial and glomerular cells, stimulate the expression of a variety of gene products that help to enhance oxygen delivery, such as VEGF and erythropoietin (EPO), and protect cells from acute ischemia, and thus would appear to exert a cytoprotective effect on renal tissues (14-16). On the other hand, recent findings by our group and others have suggested that hypoxia, through HIF-1, could enhance fibrogenesis via upregulation of connective tissue growth factor (CTGF) and factors involved in ECM modification, such as tissue inhibitor of matrix metalloproteinase 1 (TIMP-1) and plasminogen activator inhibitor 1 (PAI-1) (17-19).

An important mechanism underlying the development of tubulointerstitial fibrosis is epithelial-to-mesenchymal transition (EMT) (20-22). Hypoxic regulation of EMT has been shown to be involved 


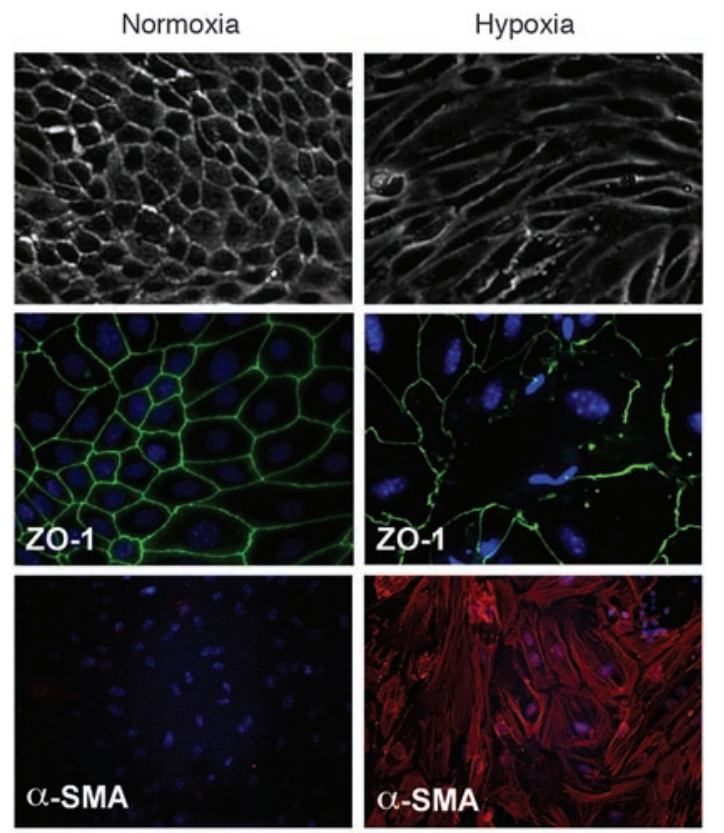

in cancer progression and metastasis $(23,24)$, and HIF-1 has been identified as a regulator of EMT in several cancer cell lines $(25,26)$. We therefore hypothesized that hypoxia through HIF-1 may promote fibrogenesis via stimulation of EMT in addition to the induction of gene products that promote ECM accumulation. During EMT, epithelial cells dedifferentiate, lose cell polarity and epithelial surface markers, induce the expression of mesenchymal markers, and display phenotypic alterations resulting in the formation of a more motile fibroblast-like cell (27). We previously reported that interstitial cells positive for fibroblast-specific protein-1 (FSP-1; also known as S100A4), which in part originate from renal epithelial cells through EMT, play an important role in the progression of renal fibrosis (20). Although a large number of molecules that contribute to the induction of EMT have been identified, little is known about the cellular stresses that trigger EMT (22).

Given the diversity of its actions, i.e., it has both cytoprotective and profibrogenic roles, it remains unclear whether activation of HIF-1 signaling exerts a beneficial or harmful effect on chronically hypoxic renal tissues. In this report, we combine experimental studies using primary cell culture and conditional knockout mice with clinical data from renal biopsy tissues to assess and dissect the role of epithelial HIF-1 and its target genes in renal fibrogenesis. Here we show that HIF-1 enhances EMT in renal epithelial cells in vitro and that genetic ablation of epithelial Hif1a in vivo results in a reduction of fibrosis in the unilateral ureteral obstruction (UUO) model. Clinically, the extent of HIF-1 $\alpha$ expression was associated with the degree of fibrotic injury in renal biopsy tissues from patients with CKD. Furthermore, we found that the expression of HIF target lysyl oxidase-like 2 (LOXL2) is increased in CKD patients and demonstrate experimentally that lysyl oxidase genes play a role in the regulation of epithelial cell motility in vitro and the development of fibrosis in vivo. Thus, our studies provide clinical and genetic evidence that HIF- $1 \alpha$ is stabilized in progressive renal disease and that activation of HIF-1 may promote fibrosis by increasing expression of ECMmodifying factors and lysyl oxidases and by enhancing transition of epithelial cells toward a mesenchymal phenotype.

\section{Figure 1}

Hypoxia induces morphological and phenotypic changes in PTECs consistent with an EMT phenotype. PTECs cultured under hypoxic ( $1 \% \mathrm{O}_{2}$; right panels) compared with normoxic $\left(21 \% \mathrm{O}_{2}\right.$; left panels) conditions displayed altered cell shape (phase contrast, original magnification, $\times 100)$, reduced staining for the epithelial junction protein ZO-1 (green; original magnification, $\times 400$ ), and increased staining for $\alpha$-SMA (red; original magnification, $\times 200$ ), nuclei were stained with DAPI (blue).

\section{Results}

Hypoxic stimulation induces a mesenchymal phenotype in epithelial cells consistent with EMT through Hif-1-dependent and-independent mechanisms. Epithelial cells undergoing EMT typically lose expression of epithelial markers such as ZO-1 and E-cadherin and gain expression of mesenchymal markers including $\alpha$-SMA and vimentin. In addition, morphological changes occur, and transitioned epithelial cells lose their polarity and become elongated and more fibroblast-like (22). We found that murine primary tubular epithelial cells (PTECs) cultured under hypoxic conditions $\left(1 \% \mathrm{O}_{2}\right)(\mathrm{a})$ altered their morphology and appeared more elongated and had (b) reduced expression of the epithelial junction protein ZO-1 and (c) increased expression of $\alpha$-SMA when compared with normoxic PTECs (Figure 1), features that are consistent with epithelial cells undergoing EMT to produce a more fibroblast-like cell type. We hypothesized that hypoxia could play a role in the development of renal fibrosis through stimulation of the EMT process.

We have previously shown that PTECs stabilize Hif- $1 \alpha$ and not Hif- $2 \alpha$ protein in response to hypoxia (17). To assess the contribution of Hif-1 to the hypoxic induction of EMT, we examined FSP-1 protein as a marker of EMT in $\mathrm{Hifla}^{+/+}$and Hif1 $\mathrm{a}^{-/-}$PTECs exposed to hypoxia for 1-5 days. PTECs were isolated from mice homozygous for the wild-type Hif1 a allele and expressing the ROSA26RlacZ reporter along with $P E P C K$-cre, which targets the majority of proximal tubular epithelial cells (Hif1 $\mathrm{a}^{+/+} ;$ROSA26RlacZ;PEPCK-cre, referred to as $\mathrm{Hifl}^{+/+}$); or from mice homozygous for the conditional Hif1a 2-lox allele and expressing ROSA26RlacZ together with PEPCK-cre (Hif1 $a^{2 l o x / 2 l o x} ;$ ROSA26RlacZ;PEPCK-cre, referred to as Hif1 $\left.\mathrm{a}^{-/}\right)(28,29)$. As FSP-1 is expressed in both fibroblasts and transitioning epithelia, we utilized the ROSA26RlacZ reporter transgene (30) to tag epithelial cells in primary cultures, allowing us to distinguish contaminating FSP-1-expressing fibroblasts from epithelial cells that have undergone EMT and express de novo FSP-1. Epithelial cells that express PEPCK-cre activity lose the STOP cassette through recombination of the loxP sites and express functional lacZ (Figure 2A). With this experimental system, we were able to identify FSP-1-expressing cells that originated from an epithelial lineage (Figure 2A). A significant increase in the percentage of FSP-1-positive epithelial cells was observed in $\mathrm{Hifla}^{+/+}$PTECs exposed to hypoxia for 5 days (Figure 2B; from $4.9 \% \pm 0.9 \%$ to $10.1 \% \pm 1.0 \% ; P<0.01)$. In contrast, Hifl $a^{-/}$PTECs did not display an increase in FSP-1-positive cells in response to hypoxia at any time point analyzed. Similar results were found in Hif $1 \mathrm{a}^{+/+}$PTECs that were lacZ tagged using the $\gamma$-GT-cre transgene, which is expressed in cortical proximal tubular epithelial cells (20) (data not shown). Taken together, these findings suggest that hypoxia increases the number of FSP-1-positive cells in PTEC cultures in a HIF-1-dependent fashion. Given that FSP-1 mRNA did not show the hypoxic increase that would be expected from a HIF target gene (data not shown) and that FSP-1 protein levels were 

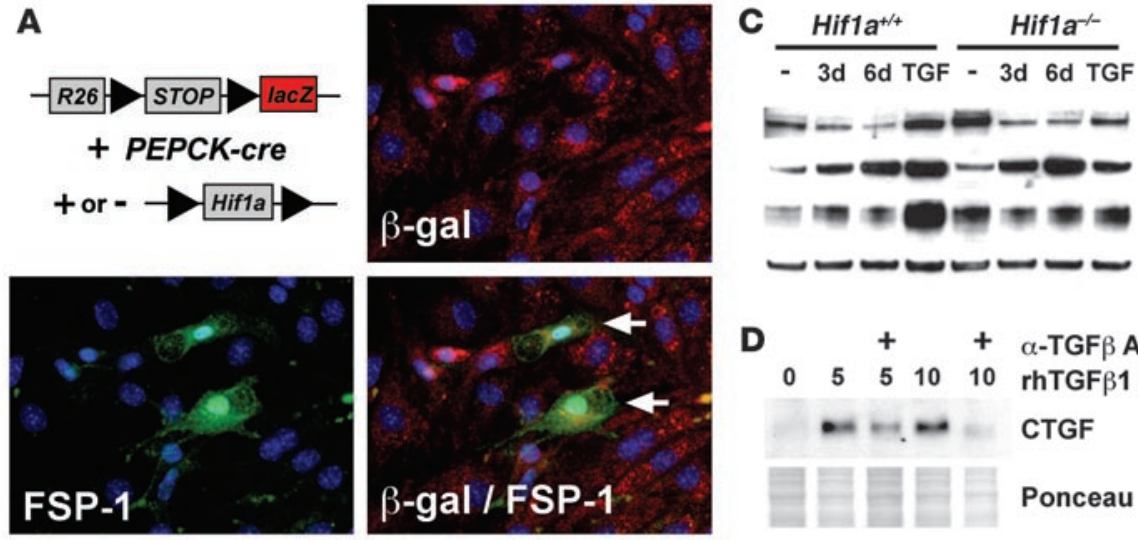

Hypoxia

ZO-1

$\alpha$-SMA

CTGF
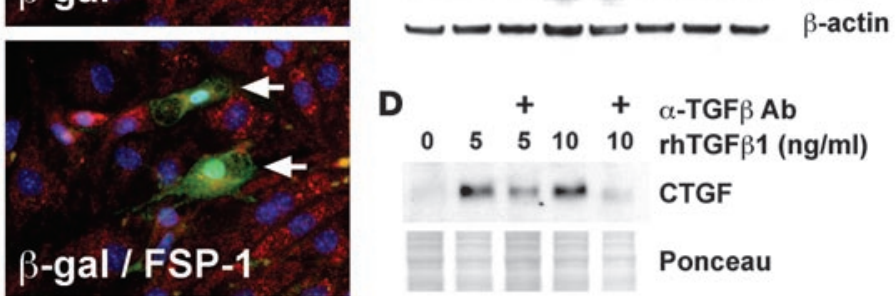

D

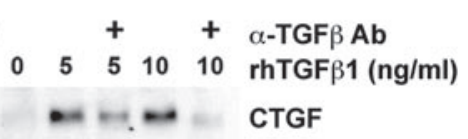

Ponceau
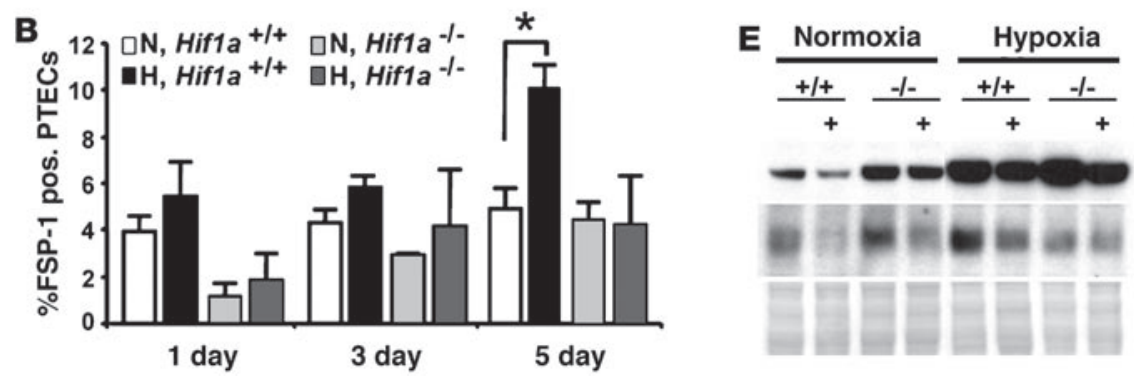

Hif1a

$\alpha-$ TGF $\beta$ Ab

$\alpha$-SMA

CTGF

Ponceau

\section{Figure 2}

Hif-1 promotes EMT in PTECs. (A) Upper-left panel: Schematic illustrating the genetic make-up of mice used for the in vitro EMT studies. Mice expressed the ROSA26RLacZ reporter (top) in conjunction with the PEPCK-cre transgene, in the presence (+) or absence (-) of the floxed Hif1a conditional allele (bottom). Triangles indicate the presence of loxP sites. Hif1 $\mathrm{a}^{+/+}$or Hif1 $\mathrm{a}^{-/}$PTECs were cultured under normoxia or hypoxia for 0-5 days. Cells were stained for $\beta$-gal (red) and the mesenchymal marker FSP-1 (green; original magnification, $\times 400$ ). Epithelial cells undergoing EMT stained both red and green (arrows). (B) Percent FSP-1-positive epithelial cells in Hif1 $\mathrm{a}^{+/+}$or Hif1 $\mathrm{a}^{-/-}$cultures exposed to normoxia $(\mathrm{N})$ or hypoxia $(\mathrm{H})$ for 1-5 days. Scale bars represent mean values \pm SEM. ${ }^{*} P<0.01$ (C) Western blot analysis for ZO-1, $\alpha-S M A$, and CTGF in Hif1 $\mathrm{a}^{+/+}$or Hif1 $\mathrm{a}^{-/-}$PTECs exposed to normoxia or hypoxia for 3 or 6 days. PTECs were stimulated with $3 \mathrm{ng} / \mathrm{ml}$ TGF- $\beta 1$ for 3 days as a positive control for $\alpha$-SMA and CTGF induction. $\beta$-Actin is included as loading control. (D) Neutralizing Ab against TGF- $\beta$ ( $\alpha$-TGF $\beta$ Ab) $(1 \mu \mathrm{g} / \mathrm{ml})$ inhibits TGF- $\beta 1$ induction of CTGF in PTECs. (E) Western blot analysis for $\alpha$-SMA and CTGF in Hif1 $\mathrm{a}^{+/+}$or $\mathrm{Hifl}^{-/-}$PTECs cultured for 6 days under either normoxia or hypoxia in the absence or presence $(+)$ of neutralizing Ab against TGF- $\beta$.

only elevated after prolonged exposure to hypoxia (greater than 48 hours), we interpret the observed increase in FSP-1-expressing cells therefore as a consequence of EMT.

We next examined whether Hif-1 is directly involved in the upregulation of $\alpha$-SMA and the decrease in ZO-1 in PTECs after 3 or 6 days treatment with hypoxia $\left(1 \% \mathrm{O}_{2}\right)$. For these studies, cells were isolated from $H i f 1 a^{2 l o x / 2 l o x} ; R 26-r t T A 2 ; L c-1$-cre mice, which ubiquitously express a tetracycline-inducible cre-recombinase. Hifla deletion was induced upon exposure to $2 \mu \mathrm{g} / \mathrm{ml}$ doxycycline for 48 hours after isolation, as previously described (doxycyclinetreated cells are hereafter referred to as $\mathrm{Hifl}^{-/-}$; untreated cells as $\mathrm{Hifl}^{+/++}$(17). We observed induction of $\alpha$-SMA protein in both $\mathrm{Hifla}^{+/+}$and Hif1 $\mathrm{a}^{-/-}$PTECs (Figure 2C), suggesting that Hif-1 was not involved in hypoxic induction of $\alpha$-SMA in PTECs. Similarly, the loss of ZO-1 expression in response to hypoxia was observed in both $\mathrm{Hifla}^{+/+}$and $\mathrm{Hifla}^{-/-}$cells, suggesting that Hif- $1 \alpha$ is not required for the downregulation of ZO-1 (Figure 2C). Enhanced signaling through TGF- $\beta 1$, a well-known inducer of EMT, has been observed in response to hypoxia $(21,22)$. In order to determine whether the hypoxic induction of $\alpha$-SMA is TGF- $\beta 1$ dependent, we (Figure 3A). In both normoxic Hifla $a^{+/}$and Hif1 $a^{-/-}$PTECs, the scratch was still apparent by 24 hours, whereas hypoxic $H i f 1 a^{+/+}$ PTECs completely migrated across the scratch by 24 hours (hypoxic Hifla $\mathrm{a}^{++}$PTECs displayed a 2.4-fold increase in migration compared with normoxic Hif1a ${ }^{+/+}$PTECs; $\left.P<0.05\right)$. In contrast, hypoxic Hifla $1-$ PTECs failed to migrate across the scratch by 24 hours and did not show a significant increase in migration compared with normoxic Hifla $a^{-/}$PTECs (Figure 3A). We conclude therefore that hypoxia can enhance epithelial cell migration in a Hif-1-dependent manner.

It has recently been shown that hypoxia can enhance migration in cancer cells through HIF-1-dependent upregulation of lysyl oxidase activity (23). We analyzed the hypoxic induction of lysyl oxidase (Lox) mRNA and the related family member lysyl oxidase-like 2 (LoxL2) in Hif1a $a^{+/+}$and Hifla $a^{-/}$PTECs by quantitative real-time PCR (Figure 3B). To ensure complete inactivation of the Hif- 1 transcription factor, we monitored the expression of 4 Hif- 1 targets, Pgk, Vegf, Mdr-1, and Pai-1, the latter encoding a fibrosis-promoting modulator of ECM and EMT $(32,33)$. All 4 Hif-1 target genes were hypoxia inducible in $\mathrm{Hifla}^{+/+}$PTECs, but not in 

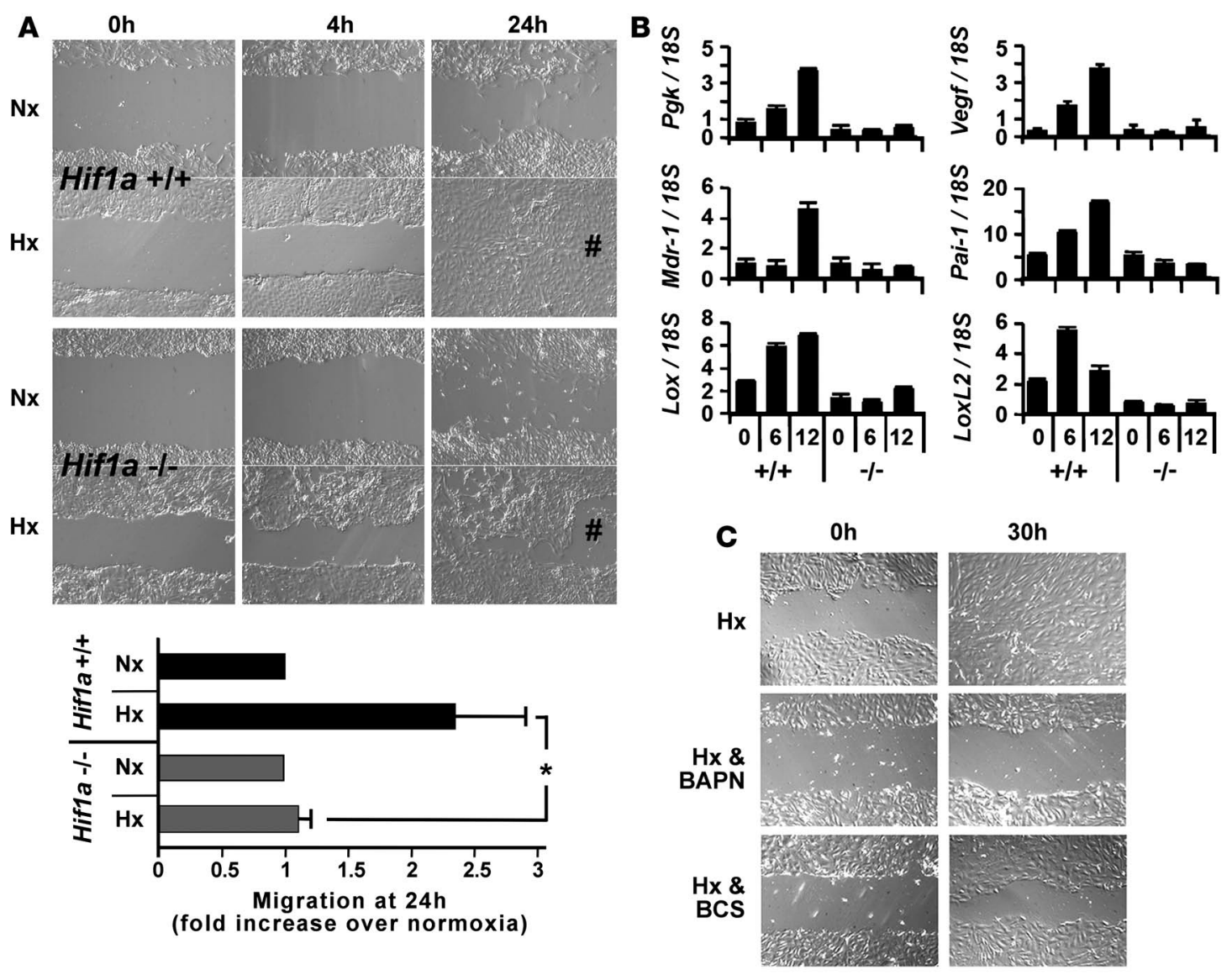

Figure 3

Hif-1 enhances epithelial cell migration through induction of lysyl oxidases. (A) Hif1 $\mathrm{a}^{+/+}$or Hif1 $\mathrm{a}^{-/-}$PTECs were cultured under normoxia or hypoxia for 6 days. Migration was analyzed by introducing a scratch into the epithelial layer and measuring scratch width, i.e., migration distance, at 0,4 , and 24 hours. Hypoxic Hif1 $a^{+/+}$cells (top panel) displayed complete scratch closure after 24 hours, whereas the scratch was still apparent in hypoxic Hif1 $a^{-/-}$cells (lower panel); compare images labeled with \#. Original magnification, $\times 100$. Graph shows fold increase of hypoxic (Hx) $\mathrm{Hif1}^{+/+}$(black bars) and Hif1 $\mathrm{a}^{-/-}$(gray bars) PTECs compared with their respective normoxic $(\mathrm{Nx})$ migration. ${ }^{*} P<0.05$. (B) Quantitative real-time PCR analysis of Pgk (4.2-fold induction at 12 hours), Vegf (13.8-fold increase at 12 hours), Mdr-1 (4.6-fold increase), Pai-1 (3.2-fold induction at 12 hours), Lox and LoxL2 (2.4- and 2.5-fold induction at 12 and 6 hours, respectively) mRNA in Hif1 $\mathrm{a}^{+/+}(+/+)$or Hif1 $\mathrm{a}^{-/-}(-/-)$PTECs exposed to hypoxia for 0, 6, and 12 hours. Gene expression was normalized to $18 \mathrm{~S}$ mRNA. (C) Hif1 $\mathrm{a}^{+/+}$PTECs were cultured under hypoxia for 6 days; 2 hours prior to wounding, lysyl oxidase inhibitors BAPN or BCS were added and cell migration monitored over 30 hours. Hx, untreated control cells (hypoxia without inhibitor). Original magnification, $\times 100$.

$\mathrm{Hifl}^{-/-}$cells, which is consistent with highly efficient recombination of Hifla in response to doxycycline treatment. Similarly, both Lox and LoxL2 mRNA were induced by hypoxia in $\mathrm{Hifl}^{+/+}$(a 2.4fold induction for Lox at 12 hours and a 2.5 -fold induction for LoxL2 at 6 hours; $P<0.05$ ) and not in Hif1 $\mathrm{a}^{-/-}$PTECs, suggesting a requirement for Hif-1 in the hypoxic induction of lysyl oxidases. Lysyl oxidases are copper-dependent enzymes with intracellular and extracellular activities that catalyze the oxidation of lysine residues in collagen and elastin and can modulate cell migration $(34,35)$. Addition of lysyl oxidase inhibitors $\beta$-aminopropionitrile (BAPN) or bathocuproine disulfonate (BCS) attenuated hypoxiaenhanced migration of Hif1 $\mathrm{a}^{+/+}$PTECs (Figure 3C). In hypoxic cells, complete scratch closure was observed by 24 hours (Figure $3 \mathrm{~A}$ ); however, when hypoxic cells were pretreated with either BAPN or BCS for 2 hours, migration was inhibited so that the scratch was still apparent for up to 30 hours. BAPN is an irreversible inhibitor of lysyl oxidases and inhibits all lysyl oxidase family members.
BCS is a membrane-impermeable copper chelator that inhibits extracellular lysyl oxidase activity. In combination, these data suggest that hypoxic enhancement of epithelial migration, a feature of transitioned epithelial cells, involves Hif-1-mediated induction of lysyl oxidases.

Renal tubular bypoxia following UUO. Collectively, we have shown in an in vitro system that hypoxia can induce morphological and functional changes consistent with EMT. We have also determined that Hif- 1 , although not required for increased $\alpha$-SMA expression or loss of ZO-1 expression, is required for enhanced migration through increased lysyl oxidase activity. Therefore, we hypothesized that Hif-1 may play a role in promoting renal fibrosis in vivo through enhancement of EMT and employed murine UUO, in which ligation of the ureter causes blockage of urine flow through the kidney and induction of fibrosis. We first determined whether kidney tissues experienced a pathological deficit of oxygen during the development of renal fibrosis. Oxygen tensions in the renal 
A
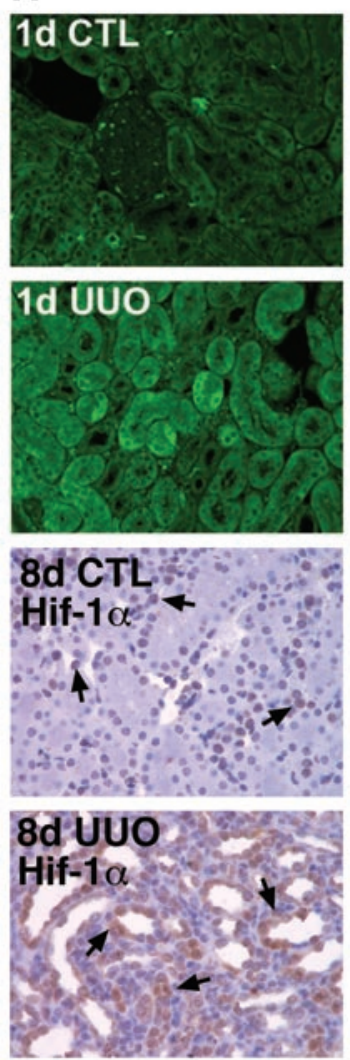
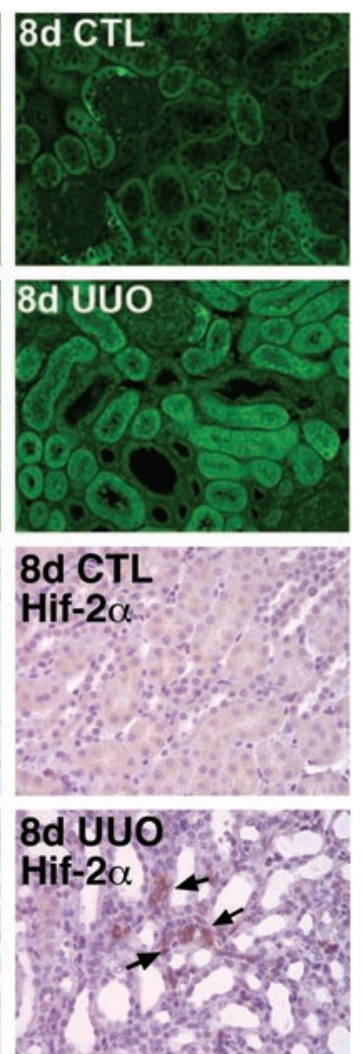

B
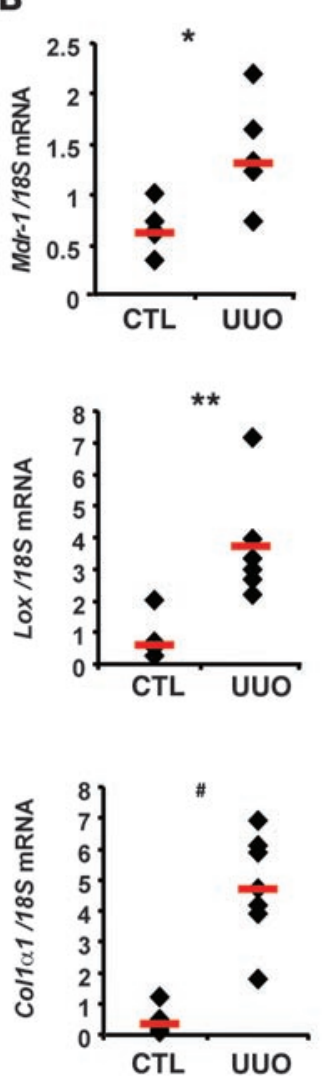
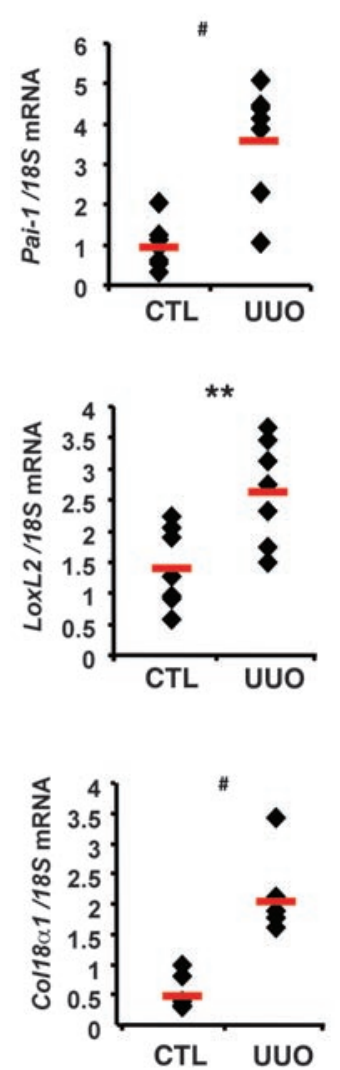

Figure 4

UUO kidneys are hypoxic prior to development of tubulointerstitial fibrosis. (A) Hypoxyprobe (Chemicon) was used to detect hypoxic regions in obstructed kidneys (UUO) 1 day and 8 days after ligation of the ureter (upper 4 panels). In contrast, hypoxyprobe staining was not detected in contralateral kidneys (CTL) 1 day after ligation but was apparent at low levels by day 8; original magnification $\times 200$. Lower panels show cortical immunostaining for Hif- $1 \alpha$ and Hif-2 $\alpha$ in 8-day UUO and CTL kidneys; original magnification, $\times 400$; arrows indicate cells with positive nuclear

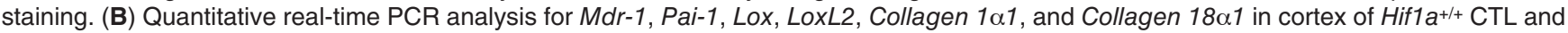
UUO kidneys 8 days after ligation. Shown are relative expression values normalized to $18 S$ rRNA. Data points represent individual kidneys; red bars represent mean values; $n=7 ;{ }^{*} P<0.05,{ }^{* \star} P<0.01$, ${ }^{\#} P<0.001$.

cortex can vary greatly but are estimated at $5 \% \mathrm{O}_{2}$ (equivalent to approximately $38 \mathrm{mmHg}$ ), with approximately $1 \%-2 \% \mathrm{O}_{2}(8-15$ $\mathrm{mmHg}$ ) in the renal medulla. Although HIF- $\alpha$ stabilization can occur at oxygen tensions lower than $5 \% \mathrm{O}_{2}$, HIF proteins are typically not detectable unless a substantial decrease in oxygen supply has occurred (e.g., as a result of radiocontrast exposure or carbon monoxide poisoning) or when HIF- $\alpha$ degradation is inhibited pharmacologically (e.g., by cobalt chloride) $(36,37)$. In the UUO model, tubulointerstitial fibrosis becomes evident 4 days after ligation and progresses so that by day 8 , renal tissue is characterized by marked expansion of the interstitial area due to increased fibroblast numbers, enhanced ECM deposition, and increased mononuclear cell infiltration, which is associated with tubular atrophy and apoptosis (38). Tissue hypoxia was detected using pimonidazole hydrochloride, which forms protein adducts in cells that experience an oxygen level of approximately $1 \% \mathrm{O}_{2}$. Pimonidazole staining revealed that subcortical tubular epithelial cells in UUO kidneys experienced a discrepancy between oxygen supply and demand as early as 24 hours after ligation (Figure 4A; 1d UUO), which was sustained throughout the development of fibrosis (Figure 4A; 8d UUO). In contrast, contralateral kidneys on day 1 after ligation did not show substantial evidence of hypoxia in this assay, whereas a low level of hypoxia was detected in subcortical tubular epithelial cells on day 8 . We monitored the stabilization of Hif- $1 \alpha$ and Hif- $2 \alpha$ protein in contralateral and UUO kidney cortices at days 1 and 8 after ligation by immunohistochemistry and Western blot analysis. Although Hif- $1 \alpha$ protein was detectable by immunohistochemistry in renal epithelial cells on days 1 and 8 (Figure 4A), we were unable to detect Hif- $1 \alpha$ by Western blot analysis, which was most likely due to technical difficulties (28). Hif- $2 \alpha$ was detected by immunoblot as early as day 1 (11.7 increase on day 1 compared with sham-operated kidneys and 10.7-fold increase by day 8 after UUO) (Supplemental Figure 1; supplemental material available online with this article; doi:10.1172/JCI30487DS1). Interestingly, contralateral kidneys revealed an increase in Hif- $2 \alpha$ levels as well (up to 5-fold) compared with sham-operated kidneys and showed evidence of Hif- $1 \alpha$ stabilization by immunohistochemistry (Figure $4 \mathrm{~A})$. The detection of Hif- $\alpha$ in the contralateral kidney may be a reflection of enhanced oxygen demand due to augmented work load, as the nonobstructed kidney compensates for the lack of renal function in the obstructed kidney. Consistent with increased Hif- $\alpha$ expression is the increased expression of Hif target genes such as $M d r-1$, 
A
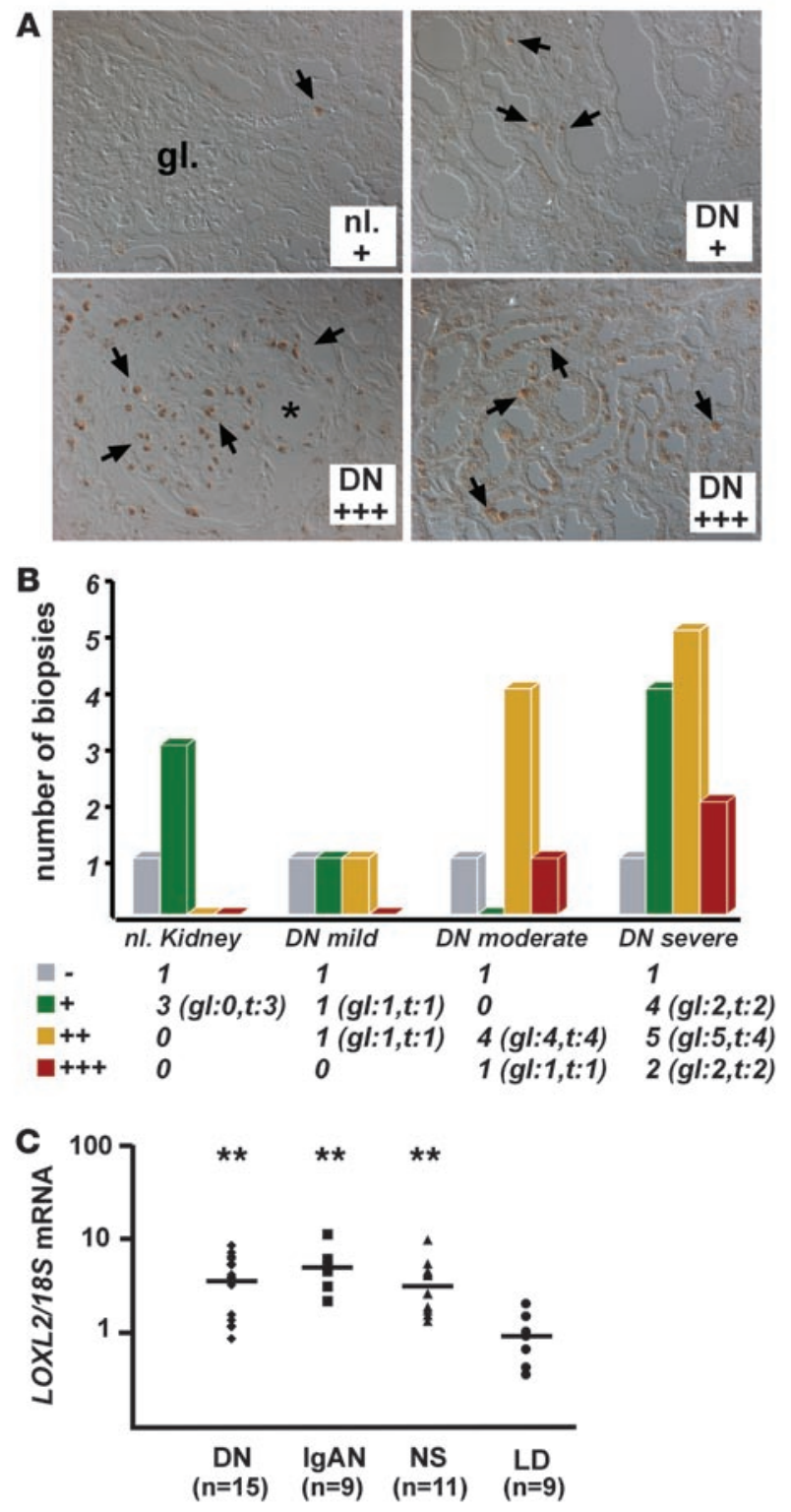

Pai-1, Lox, and LoxL2 $(19,23,39,40)$ that we found in kidneys on day 8 after UUO compared with contralateral kidneys (Figure 4B). In addition, we observed upregulation of Collagen $1 \alpha 1$ and Collagen $18 \alpha 1$ in response to ureteral ligation, which is consistent with fibrosis development in our model (18). Taken together, these results suggest that UUO kidneys are hypoxic and display protein and gene expression changes consistent with Hif activation.

Detection of HIF-1 $\alpha$ in renal tissue samples from patients with CKD. After demonstrating that HIF-1 is activated in a mouse model of renal fibrosis, we next examined the extent of HIF-1 $\alpha$ stabilization in a recently published series of archival renal biopsy tissues from patients with diabetic nephropathy (DN) that had different degrees of tubulointerstitial injury (41). DN accounts for $44 \%$ of newly diagnosed end-stage renal disease cases in the United States and therefore represents a major portion of progressive kidney diseases (42). Paraffin-embedded renal tissues from 21 patients with DN and 4 normal kidneys were examined for HIF- $1 \alpha$ expression using a previously described detection method (43). Tissue

\section{Figure 5}

HIF- $1 \alpha$ in renal biopsies from patients with CKD. (A) HIF- $1 \alpha$ immunostaining in formalin-fixed, paraffin-embedded renal biopsy tissues from patients with DN analyzed by differential interference contrast microscopy. Top row: Tissue from a normal control kidney (nl.) and from a DN kidney, both with $1+$ staining ( $\leq 25 \%$ cells positive per visual field). Bottom row: Representative photographs from DN kidneys with $3+$ staining ( $>50 \%$ cells stained positive). A glomerulus ( $\mathrm{gl}$.) with HIF- $1 \alpha$-positive cells is shown on the left; the tubulointerstitial compartment from a different DN kidney is shown on the right. Arrows highlight cells with nuclear HIF- $1 \alpha$ staining. The asterisk indicates area with nodular sclerosis. (B) Summary of HIF-1 $\alpha$ expression analysis in DN. DN cases are grouped according to tubulointerstitial injury score as described by Hohenstein et al. (41). The number of biopsies with glomerular or tubular staining $(\mathrm{t})$ is shown in parentheses. -, absence of staining;,$+ 1 \%-25 \%$ of cells per visual field with positive staining; ,$++>25 \%-50 \% ;+++,>50 \%$ of cells with positive staining. (C) Expression analysis of LOXL2 in microdissected tubulointerstitium from patients with DN, IgA nephropathy (IgAN), and hypertensive nephrosclerosis (NS) by real-time PCR. Shown are relative expression values normalized to $18 S$. Pretransplant biopsies from living donor kidneys (LD) were used as control. ${ }^{* *} P<0.01$ by Mann-Whitney $U$ test.

sections were analyzed for cellular sublocalization of staining and scored based on the percentage of HIF- $1 \alpha$-expressing cells, with $3+$ being the highest score ( $>50 \%$ of all cells positive). While little positive staining for HIF- $1 \alpha$ was found in the renal tubules of normal kidneys (HIF- $1 \alpha$ score of $\leq 1$ and absent in glomeruli; Figure 5A), prominent HIF-1 $\alpha$ staining ( $>25 \%$ of all cells positive) was found in 13 of 21 patients with DN (HIF- $1 \alpha$ score of $\geq 2, P=0.039$ compared with normal controls, when grouped by score of $\geq 2$ or $<2$; Fisher's exact test; Figure 5B). HIF-1 $\alpha$ expression was also found in fibrotic areas of renal tissues from patients with IgA nephropathy (Supplemental Figure 2). Microarray analysis of human biopsy material suggested that HIF target genes are differentially regulated in microdissected renal tubulointerstitial tissues from patients with DN (Supplemental Figure 3). Genes that were found to be upregulated included PGK-1, CXCR4, LOXL2, and phosphofructokinase (PFKFB3). Increased expression was confirmed by real-time PCR analysis for LOXL2 $(P<0.01$; Figure $5 C)$ in a separate set of patients with DN. A comparable increase in LOXL2 expression levels was also found in microdissected tubulointerstitial compartments of renal biopsy tissues from patients with IgA nephropathy and hypertensive nephrosclerosis, indicating that increased LOXL2 expression is associated with the development of CKD in general and is not restricted to DN alone.

Hif-1 $\alpha$ deletion in UUO kidneys impairs development of fibrosis. Since we detected hypoxia in tubular epithelia and the hypoxic response in renal tubular epithelial cells is mediated by Hif- 1 and not Hif-2 $(17,37)$, we hypothesized that inactivation of Hif- $1 \alpha$ in these cells may attenuate the ability of hypoxia to enhance renal fibrosis. We

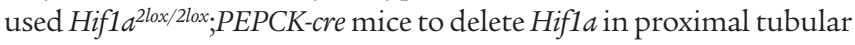
epithelial cells in vivo (referred to as mutant) and compared these with cre-negative control mice. PEPCK-cre recombination occurred in approximately $50 \%-70 \%$ of proximal tubular epithelial cells as determined with the ROSA26RlacZ cre-reporter (Supplemental Figure 1). Because of the potential limitations of this approach due to the presence of endogenous lac $Z$ activity in renal tubules, we determined mRNA levels of the recombined Hifla exon 2 by quantitative RT-PCR and found a reduction of approximately $25 \%$ compared with control (Supplemental Figure 1). This reduc- 


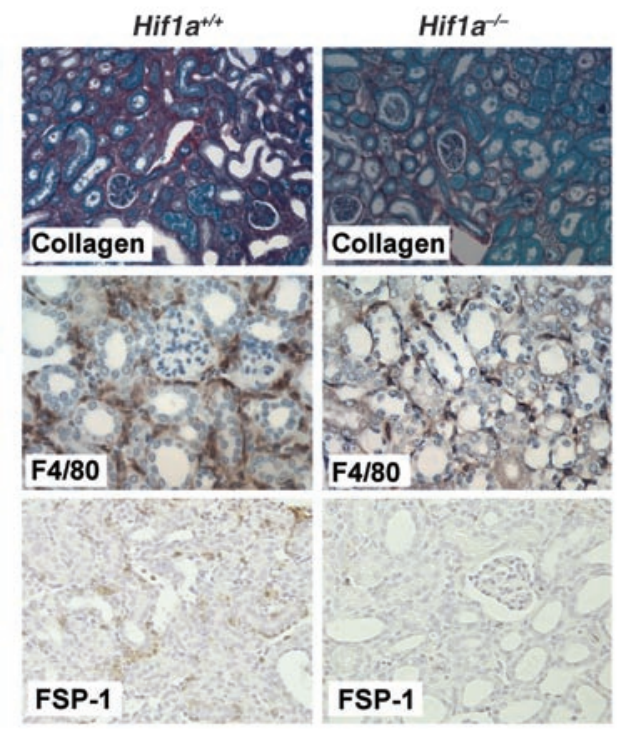

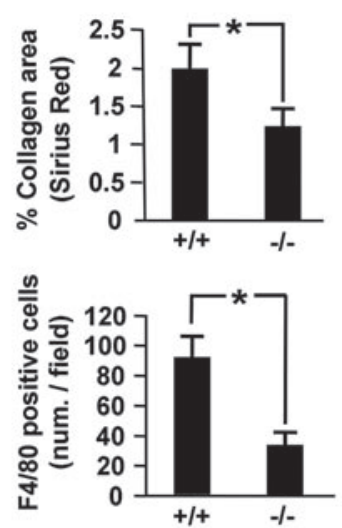

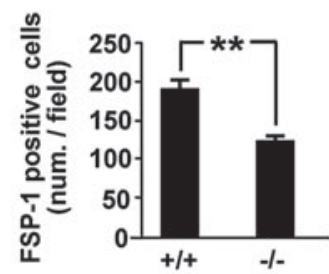

\section{Figure 6}

Deletion of Hif1a in PTECs attenuates renal fibrogenesis. $\mathrm{Hif1}^{+/+}$and Hif1 $\mathrm{a}^{-/-}$kidneys were stained for collagen content (sirius red staining of collagen fibers shown in red; original magnification, $\times 200 ; n=8$ for mutant and $n=7$ for control), macrophage marker $\mathrm{F} 4 / 80$ (original magnification, $\times 400 ; n=3$ in each group), and EMT marker FSP-1 (original magnification, $\times 400 ; n=9$ for mutant and $n=5$ for control). For statistical analysis, sirius red-positive areas from 10 individual measurements per mouse were averaged across control and mutant cohorts. Morphometric analysis showed a reduction of all 3 stains in Hif1a mutant tissues. Scale bars represent mean values \pm SEM; ${ }^{\star} P<0.05,{ }^{* \star} P<0.01$. tion was ablated in UUO kidneys 8 days after ligation, as Hifla mRNA levels were similar in control and mutant mice, possibly due to increased Hifla mRNA expression in nonrecombined cells, and warrants further investigation. Therefore, the analysis of Hif-1 target gene expression in mutant kidneys was noninformative in day 8 UUO kidneys, limiting our investigation of fibrogenesis in UUO kidneys to morphometric and histological studies.

Collagen staining was performed on 7 control and 8 mutant mice 8 days after ureteral ligation (Figure 6). Although serum creatinine was not different between groups, sirius red staining and morphometric analysis revealed an approximately $40 \%$ reduction in the amount of interstitial collagen deposition in Hif- 1 a mutants $(1.99 \% \pm 0.30 \%$ to $1.23 \% \pm 0.22 \%, P<0.05$, when averaged across all mutant UUO kidneys). Similarly, staining for F4/80, a macrophage marker, showed an approximately $60 \%$ reduction in mutant kidneys, suggesting reduced inflammation (from $91.53 \pm 16.30$ to $32.64 \pm 8.52$ cells per visual field; $n=3 ; P<0.05$ ). Furthermore, the number of FSP-1-positive cells in mutant UUO kidneys was significantly reduced compared with control (from $191 \pm 15.60$ $[n=5]$ to $123 \pm 7.31[n=9]$ cells per visual field; $P<0.01)$. These data suggest that the expression of Hif- $1 \alpha$ is associated with increased tubulointerstitial injury and fibrosis in vivo.

Since lysyl oxidases have been implicated in EMT and mediate hypoxia-stimulated epithelial cell motility (Figure 2), we investigated whether

\section{Figure 7}

Inhibition of lysyl oxidases reduces fibrosis in UUO kidneys. Shown are the number of FSP-1positive cells (top panel) and the area stained for collagen by sirius red (bottom panel) in vehicletreated (VEH-UUO) and BAPN-treated UUO (BAPN-UUO) kidneys; original magnification, $\times 100$ (top panel) and $\times 400$ (bottom panel). Scale bars represent mean values \pm SEM; ${ }^{*} P<0.05$, ${ }^{* *} P<0.001 ; n=6$. lysyl oxidase inhibition in vivo would affect the development of fibrosis in UUO kidneys. We found that Hif-1-competent mice treated with BAPN and subjected to UUO showed a reduction in FSP-1-positive renal interstitial cells (46\%, from $113.43 \pm 16.19$ cells per field to $62.49 \pm 4.04 ; n=6 ; P<0.05)$. This was associated with a significant reduction in collagen accumulation $(35 \%$ reduction in collagen staining, from $8.80 \% \pm 0.61 \%$ to $5.71 \% \pm 0.48 \%$; $n=6$; $P<0.001$ ) compared with vehicle-treated controls (Figure 7), whereas F4/80 cell numbers were not different between groups $(15.8 \pm 4.00$ vs. $15.31 \pm 5.09$ cells per visual field; $n=3$ ). Taken together, these data suggest that lysyl oxidases play a role in the development of renal fibrosis and thus may mediate HIF-1's profibrotic effects.

\section{Discussion}

The presence of hypoxia has been detected in several renal disease models and has been attributed to capillary rarefaction with subsequent reduction in blood perfusion. This pathological feature is present in experimental obstructive nephropathy and has been frequently observed in human CKD $(1,2,44)$. Although it has been proposed that hypoxia by itself can be profibrogenic, the underly-

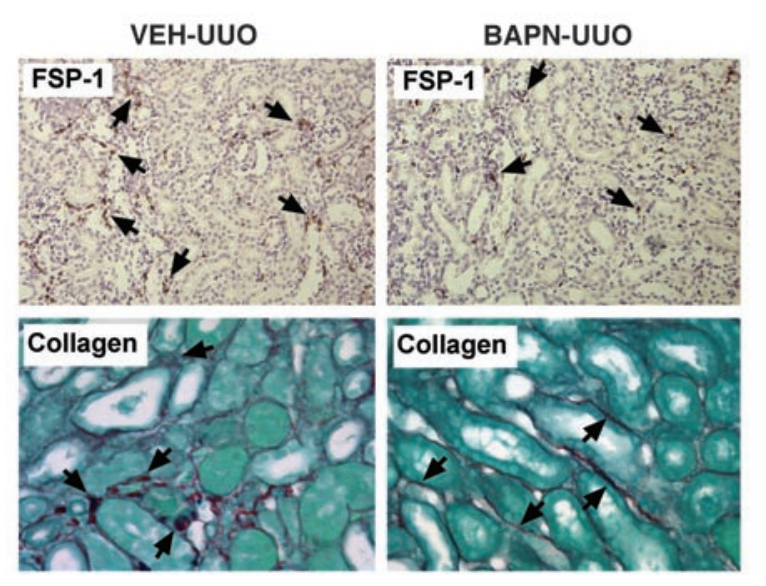

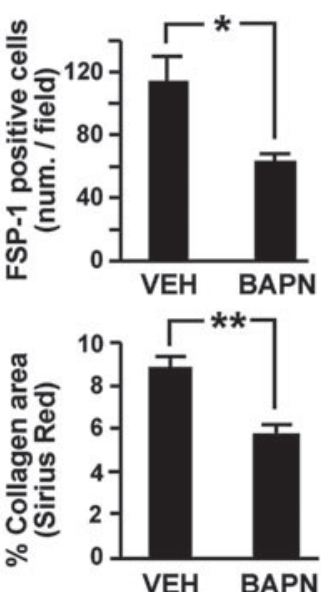




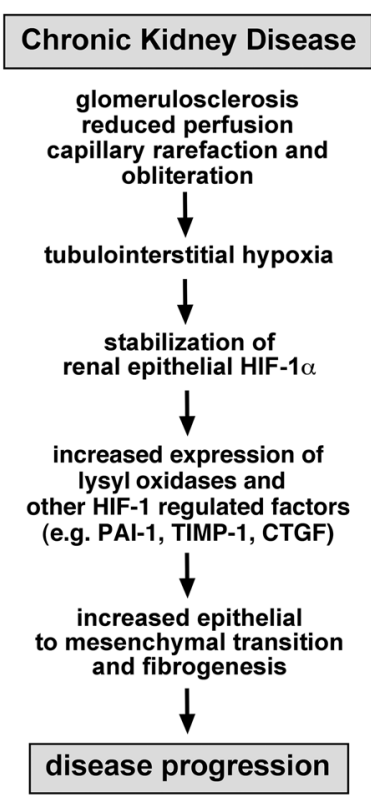

\section{Figure 8}

Model proposing a role for HIF-1 in the progression of CKD. Tubulointerstitial hypoxia is due to glomerulosclerosis and capillary rarefaction, which is commonly found in kidneys from patients with CKD. As a consequence of hypoxia in renal epithelial cells HIF- $1 \alpha$ is stabilized, resulting in increased expression of lysyl oxidase genes and other profibrogenic factors, thus promoting EMT and the accumulation of ECM.

ing molecular mechanisms remain unclear. In this report, we dissected the role of hypoxic signaling through HIF-1 in the development of fibrosis in vitro and in vivo using Cre-loxP-mediated gene targeting. We found that activation of renal epithelial Hif- $1 \alpha$ promoted fibrogenesis through modulation of EMT, whereas genetic ablation of Hif- $1 \alpha$ was protective. The clinical relevance of these findings is underscored by the extent of HIF- $1 \alpha$ expression in human renal tissue with significant tubulointerstitial injury.

Our data suggest that HIF-1 may exert its profibrotic effect through upregulation of lysyl oxidases. This notion is based on the findings that hypoxia increased Lox and LoxL2 mRNA in murine primary renal epithelial cells in a HIF-1-dependent manner, that Lox and LoxL2 were significantly increased in UUO kidneys, and that pharmacological blockade of lysyl oxidases phenocopied the effects of Hif1a deletion on hypoxia-induced cell migration in vitro and decreased renal fibrogenesis in vivo, which was associated with a substantial reduction in FSP-1-positive cells. Moreover, we found that LOXL2 expression was increased in the tubulointerstitial compartment of kidneys from patients with DN, IgA nephropathy, and hypertensive nephrosclerosis, supporting the notion that lysyl oxidases may play a role in the development and progression of renal disease. The role of lysyl oxidases in fibrogenesis appears to be general, as BAPN treatment is inhibitory in a model of liver fibrosis following bile duct ligation (45). Although initially identified by their ability to crosslink collagen and elastin fibers, lysyl oxidase and lysyl oxidase-like proteins have been shown to carry out intracellular functions as well and display a range of biological activities that extend beyond extracellular cross-linkage. These include the regulation of ras and NF- $\mathrm{KB}$ signaling, possibly through the modification of DNA-histone and histone-histone interactions (reviewed in refs. 34, 35). Recent reports indicate that LOXL2 promotes EMT through biochemical interaction with the transcriptional repressor Snail (46), which induces renal fibrosis when overexpressed in transgenic mice (47). HIF-1 induction of LOX and LOXL2 has also been shown to promote migration in human breast and cervical cancer cells and to regulate E-cadherin expression in renal cell cancer $(23,40)$. Thus, it is plausible that a HIF-1-mediated increase in the expression of lysyl oxidases in hypoxic tubular epithelial cells enables transition toward an activated fibroblast-like phenotype, migration toward the interstitium, and promotion of fibrosis (Figure 8). However, the molecular events underlying EMT are complex, and because of the transcriptional pleiotropism of HIF- 1 and the ability of its $\alpha$ subunit to interact with non-PAS domain proteins, its role in EMT via modulation of other molecular pathways, such as the Notch (48) or TGF- $\beta$ signaling pathways $(49,50)$, awaits further investigation.

A different mechanism for HIF-induced EMT appears to exist in cancer cell lines. HIF-1 has been shown to downregulate the epithelial junction protein E-cadherin, an early event in EMT, through an increase in $E$-cadherin-specific transcriptional repressors TCF3, ZFHX1A, and ZFHX1B in renal carcinoma cell lines $(25,51)$. Similarly, hypoxia increased E-cadherin repressor Snail in ovarian cancer (52). However, we found that transcriptional repressors TCF3, ZFHX1A, ZFHX1B, and Twist were decreased in hypoxic PTECs compared with normoxia (Supplemental Figure 4), which may be a result of cell type-specific differences in hypoxic gene regulation. This coincided with a HIF- $1 \alpha$-independent decrease in E-cadherin and kidney-specific cadherin (cadherin 16) transcript levels (Supplemental Figure 4). An additional feature of EMT is de novo expression of mesenchymal proteins such as $\alpha$-SMA and FSP- 1 and a loss of ZO-1 expression. In hypoxic PTECs, Hif- $1 \alpha$ was not required for hypoxic reduction of ZO- 1 or for induction of $\alpha$-SMA. Although the expression of FSP-1-positive cells increased in vitro and in vivo in a HIF-1-dependent manner, we have no evidence that FSP-1 is a direct transcriptional target of HIF and interpret these changes to be a consequence of HIF-1-regulated EMT.

We have shown that renal epithelial Hif- $1 \alpha$ is fibrogenic in a chronic renal injury model and thus exerts a disease-promoting effect. This finding is in contrast to its proposed cytoprotective role in acute and chronic ischemic injuries and suggests that HIF-1 may have a context- and/or cell type-specific biological role with regard to renal disease outcome. Preconditional activation of HIF ameliorates acute ischemic renal failure (14), and treatment of rats with the HIF- $\alpha$-stabilizing agent cobalt chloride, which inhibits prolyl-hydroxylation and prevents degradation of HIF- $\alpha$, has been shown to promote angiogenesis and to confer protection in the remnant kidney model (53) or to reduce tubulointerstitial injury in Thy- 1 nephritis through the induction of cytoprotective factors (54). Although cobalt chloride is a relatively nonspecific inhibitor of HIF- $\alpha$ degradation, these observations support the notion that stabilization of HIF- $\alpha$ prior to or at the onset of renal injury may have a beneficial effect on the progression of experimental kidney disease. It is not clear, however, which cell type or which HIF homolog, HIF- $1 \alpha$ versus HIF- $2 \alpha$, conferred protection in these models and how individual HIF targets may have modulated pathogenesis. HIF- $2 \alpha$, which under hypoxic conditions is expressed in renal interstitial, endothelial, and glomerular cells (37) and does not appear to be involved in the acute hypoxic response of renal tubular epithelial cells (17), has been 
proposed to regulate the hypoxic induction of EPO in the kidney and other tissues (55-57). More recently HIF-2 $\alpha$ has been suggested to be protective in a mouse model of acute renal ischemic injury (58), possibly through its ability to induce EPO and other cytoprotective factors. Although we found Hif- $2 \alpha$ stabilization in UUO kidneys, an increase in Epo transcript was not observed (data not shown). VEGF was found to be decreased in tubulointerstitial tissue from patients with DN, which is in contrast to other HIF-regulated genes (Supplemental Figure 3 and ref. 59). The mechanism underlying VEGF suppression in $\mathrm{DN}$ is unclear. In nondiabetic CKD, renal epithelial VEGF is increased in areas of reduced capillary density (2), and thus it is plausible that the ability of HIF transcription factors to activate certain target genes may be dependent on a specific type of disease microenvironment and its effect on HRE and/or transcriptional coactivator accessibility (reviewed in ref. 60).

The development of nonimmune, rapidly progressive glomerulonephritis and subsequent renal failure in mice with conditional inactivation of $\mathrm{PVHL}$ in podocytes illustrates the divergent biological roles of HIF in the kidney (61). Although the functional effects of PVHL gene deletion in podocytes appear to be genetic background dependent and may not be completely explained by HIF activation alone, as differences in phenotype have been reported $(62,63)$, the complexity of context- and cell type-dependent biological functions of renal HIF necessitates cell type-specific conditional knockout studies to assess the role of individual HIF transcription factors in different kidney disease models.

In summary, our study suggests that hypoxia and signaling through HIF-1 contribute to the development of interstitial fibrosis via the induction of ECM-modifying and lysyl oxidase genes and the modulation of EMT. Our findings highlight a need for careful evaluation of pharmacologic strategies that target the PHD/HIF oxygen-sensing pathway to achieve HIF- $\alpha$ stabilization and have clinical implications, as they strongly encourage therapies that aim at improving the balance between renal oxygen delivery and consumption to halt the progression of kidney fibrosis.

\section{Methods}

Generation of mouse strains. The generation, characterization, and genotyping of mouse strains used has been described previously $(17,29,30,64)$. LacZ-tagged Hifla-deficient PTECs used for in vitro analysis of EMT were isolated from Hif1 $a^{2 l o x / 2 l o x} ;$ ROSA26RlacZ;PEPCK-cre compound transgenic mice; lacZ-tagged Hifla-expressing PTECs were isolated from $\mathrm{Hifl}^{+/+}$; ROSA26RlacZ;PEPCK-cre mice and used as controls. Tetracycline-inducible Hif1a-deficient PTECs were obtained from Hif1 $a^{2 l o x / 2 l o x} ;$ R26-rtTA2;Lc-1-cre mice for the analysis of $\alpha$-SMA expression, cell migration, and HIF-regulated gene expression. Male Hif1 $a^{2 l o x / 2 l o x} ;$ PEPCK-cre mice were used for UUO analysis; cre-negative littermates served as controls. For lacZ staining, male Hif1 $1 a^{2 l o x / 2 l o x} ;$ ROSA26RlacZ; PEPCK-cre mice were used, with cre-negative littermates as controls. All procedures involving mice were performed in accordance with the NIH Guide for the Care and Use of Laboratory Animals and were approved by the University of Pennsylvania Institutional Animal Care and Use Committee.

Cell culture. PTECs were isolated from 3- to 4-week-old mice as previously described (17). Cells were cultured under normoxic conditions $\left(21 \% \mathrm{O}_{2}, 5 \%\right.$ $\mathrm{CO}_{2}, 95 \%$ humidity) until $70 \%-80 \%$ confluent and were then subjected to hypoxic conditions $\left(1 \% \mathrm{O}_{2}, 5 \% \mathrm{CO}_{2}, 95 \%\right.$ humidity) in an Invivo 2 hypoxia chamber (Ruskinn Technologies). Cells were stimulated with recombinant human TGF- $\beta 1$ (Sigma-Aldrich) in the absence or presence of anti-TGF- $\beta 1$, $-\beta 2$, and $-\beta 3$ Abs (R\&D Systems).
Scratch assay. PTECs were isolated from Hif1 $a^{2 l o x / 2 l o x} ;$ R26-rtTA2;Lc-1-cre mice expressing a tetracycline-inducible cre-recombinase transgene. After isolation, cells were cultured in the absence $\left(H i f 1 a^{+/+}\right)$or presence of $2 \mu \mathrm{g} / \mathrm{ml}$ doxycycline ( $\left.\mathrm{Hifla}^{-/-}\right)$for 48 hours (17), followed by culture under normoxic $\left(21 \% \mathrm{O}_{2}\right)$ or hypoxic $\left(1 \% \mathrm{O}_{2}\right)$ conditions for 6 days in regular growth medium until approximately $60 \%$ confluency. A scratch was introduced into the epithelial layer using a $10-\mu l$ pipette tip in triplicate wells. Six phase contrast images of each well were obtained at 0, 4, and 24 hours after wounding using a Spot2 video camera (Diagnostic Instruments); PTECs were incubated at their original oxygen tension during this period. The width of the scratch was measured at 10 random intervals for each image and averaged. Migration distance was calculated by subtracting the width at 4 or 24 hours from the original wound width at 0 hours and dividing by 2 , i.e., $(0 \mathrm{~h}-4 \mathrm{~h}$ or $24 \mathrm{~h}) / 2$. Results were displayed as fold over normoxic migration distance, where the normoxic migration distance was given the arbitrary value of 1 , and hypoxic values were normalized to their respective normoxic value. Lysyl oxidase inhibitors, $200 \mu \mathrm{M}$ BAPN or $100 \mu \mathrm{M}$ BCS (both from Sigma-Aldrich), were added 2 hours prior to wounding and were included for the duration of the assay. Scratch width was monitored over 30 hours in triplicate wells; 6 bright-field images were analyzed per well at each time point. Migration assays were performed in triplicate and repeated 3 times.

UUO. UUO was performed in 8- to 10 -week-old mice as previously described (18). In vivo hypoxia was analyzed in formalin-fixed day 1 and day 8 obstructed and contralateral kidneys using Hypoxyprobe-2 Plus Kit (Chemicon) according to the manufacturer's instructions. Contralateral kidneys were used as control. Hypoxyprobe $(60 \mathrm{mg} / \mathrm{kg})$ was injected i.p. 2 hours prior to sacrifice of mice and removal of kidneys. For in vivo lysyl oxidase inhibition studies, mice were injected daily with $100 \mathrm{mg} / \mathrm{kg}$ BAPN i.p. starting 1 day prior to UUO and continuing until 8 days after UUO, with control mice receiving saline alone.

Morphometric analysis was carried out as described below. Three of 6 BAPN-treated mice developed inflammatory infiltrates in the papilla. Occasionally focal infiltrates were seen in the outer medulla and cortex. These areas were excluded from morphometric analysis.

Kidney biopsies. Renal biopsies used for gene expression analysis were collected in a multicenter study, the European Renal cDNA Bank-KroenerFresenius Biopsy Bank (ERCB-KFB), after informed consent was obtained according to the guidelines of the respective local ethics committees, and stratified by a reference pathologist as previously described (65). Realtime PCR analysis of RNA isolated from microdissected tubulointerstitial tissue was performed on an ABI 7700 platform (Applied Biosystems) with gene-specific predeveloped assays (Applied Biosystems). For the immunohistochemical analysis of HIF- $1 \alpha$ expression, previously published archival renal tissues from core needle biopsies were used (41) with the approval of the local ethics review board.

Immunocytochemistry. $\alpha$-SMA was detected using a prediluted primary mouse $\mathrm{mAb}$ (Neomarkers) followed by Cy3-conjugated anti-mouse IgG secondary $\mathrm{Ab}$ (Vector Laboratories). ZO-1 was detected with rat anti-ZO-1 Ab (1:400) (Chemicon) followed by Alexa Fluor 488 goat anti-rat IgG (1:500) (Molecular Probes; Invitrogen). EMT in PTECs was detected using rabbit anti-FSP-1 (1:500) and biotinylated monoclonal anti- $\beta$-gal (1:100) (SigmaAldrich) followed by Cy2-conjugated donkey anti-rabbit IgG and Cy3-conjugated streptavidin (both from Jackson ImmunoResearch Laboratories Inc.). Percent EMT was calculated as the number of FSP-1-, LacZ-positive cells divided by the total number of LacZ-positive cells multiplied by 100 . Counts were taken for 10 random fields of view on triplicate wells and averaged (original magnification, $\times 400$ ). The experiment was repeated in triplicate.

Immunohistochemistry. Immunohistochemical staining was performed on UUO and contralateral kidneys from Hif1 2 2lox/2lox;ROSA26RlacZ;PEPCK-cre- 
and Hifl $1 a^{2 l o x / 2 l o x} ;$ PEPCK-cre-positive or cre-negative mice. $\beta$-Gal staining was performed on frozen tissue sections according to the protocol of MacGregor et al. (66). Collagen staining on formalin-fixed tissue sections was detected using sirius red $(0.1 \%$ fast green FCF and $0.1 \%$ direct red 80 in saturated picric acid). Ten random visual fields were analyzed per kidney section, and percent sirius red-positive area was determined using IPLab segmentation analysis (BD Biosciences). For statistical analysis, all percent areas from individual mice were averaged across control and mutant cohorts. Macrophage infiltration was assessed on formalin-fixed tissue sections using anti-F4/80 $\mathrm{Ab}$ (Abcam). EMT was monitored using anti-FSP-1 Ab (kind gift from E.G. Neilson, Vanderbilt University, Nashville, Tennessee, USA). Anti-Hif-1 $\alpha$ Ab PM16 (67) and anti-Hif-2 $\alpha$ polyclonal antiserum (64) were used for the immunohistochemical detection of Hif- $\alpha$ subunits in formalin-fixed, paraffin-embedded tissue sections from UUO and contralateral mouse kidneys. Immunostaining of HIF- $1 \alpha$ in human renal biopsy material was performed as previously described with mouse anti-human mAb clone $\alpha 67$ at a concentration of 1:10,000 (Novus Biologicals) employing a high-amplification signal detection system (CSA; Dako). Signals were analyzed with a Leica DMRB microscope using differential interference contrast. The number of HIF- $1 \alpha$-positive cells was counted in 10 randomly selected visual fields per renal biopsy (original magnification, $\times 400$ ) without knowledge of patients' diagnosis or tubulointerstitial injury score. For grading of HIF-1 $\alpha$ staining, the following scale was used: 0 : no staining; $1+: 1 \%-25 \%$ of cells per visual field stained positive; $2+$ : greater than $25 \%-50 \%$ stained positive; and $3+$ : greater than $50 \%$ of cells stained positive.

Western blot analysis. Whole-cell lysates were isolated and $20 \mu \mathrm{g}$ protein electrophoresed through 10\% PAGE gel (Novex; Invitrogen) and electrotransferred to nitrocellulose membrane (Amersham Pharmacia Biotech). Prediluted mouse monoclonal anti- $\alpha$-SMA (1:500) (Neomarkers), rabbit polyclonal anti-ZO-1 (1:2,000) (Santa Cruz Biotechnology Inc.), rabbit anti-CTGF $(1: 5,000)$ (Abcam), and anti- $\beta$-actin $(1: 5,000)$ (Sigma-Aldrich) were used as primary antibodies, followed by incubation with HRP-conjugated secondary Ab. Proteins were detected with ECL Plus Reagent (Amersham Pharmacia Biotech). Nuclear extracts for the detection of Hif- $1 \alpha$ and Hif- $2 \alpha$ were prepared as previously described (64).

Quantitative real-time PCR. Two micrograms of isolated RNA was reverse transcribed and analyzed using SYBR green or TaqMan PCR master mix. Primer sets for SYBR green analysis of murine Pgk, Vegf, and Mdr-1 were previously described (64) - Lox forward: CCACAGCATGGACGAATTCA; Lox reverse: AGCTTGCTTTGTGGCCTTCA; LoxL2 forward: GATCTTCAGCCCCGATGGA; LoxL2 reverse: CAAGGGTTGCTCTG-
GCTTGT; Hifla exon 2 forward: CAAGATCTCGGCGAAGCAA; Hifla exon 2 reverse: GGTGAGCCTCATAACAGAAGCTTT; collagen I $\alpha 1$ forward: CCCGCCGATGTCGCTAT; and collagen I 1 reverse: GCTACGCTGTTCTTGCAGTGAT; collagen $18 \alpha 1$ forward: AAGACAAGCTGACCTTCATTGACAT; collagen 18a1 reverse: GGAAGCCTCGTGGGCCTCT; Pai-1 forward: AAgACCGGAATGTGGTCTTCTCT; and Pai-1 reverse: CTGCCGCCGGGTTTTC. All genes were normalized to $18 \mathrm{~S}$ levels using the $18 \mathrm{~S}$ TaqMan set from Applied Biosystems. Samples were analyzed in triplicate, and experiments were repeated 3 times.

Statistics. Statistical analysis was performed using 2-tailed Student's $t$ test, the Fisher exact test, or the Mann-Whitney $U$ test. $P$ values of less than 0.05 were considered significant.

\section{Acknowledgments}

This work was supported by NIH grant DK072037 and in part by a University of Pennsylvania Research Foundation grant (both to V.H. Haase), the Penn Center for Molecular Studies in Digestive and Liver Diseases (P30-DK50306), the Ministry of Education and Science of Japan (grant 17590839 to M. Iwano), the Else KroenerFresenius Foundation (to C.D. Cohen), and Deutsche Forschungsgemeinschaft, Sonderforschungsbereich 423: Kidney Injury: Pathogenesis and Regenerative Mechanisms (to W.M. Bernhardt, B. Hohenstein, and K.-U. Eckardt). D.F. Higgins was supported by American Heart Association Post-Doctoral Fellowship grant 0425673U. The authors would like to thank Gary Swain and members of the Morphology Core for their technical expertise; Erinn B. Rankin, Chia H. Wu, and Travis L. Unger for technical assistance and helpful discussions; and all the members of the European Renal cDNA Bank and their patients for their support of this study (see ref. 58, for participating centers at time of study).

Received for publication September 27, 2006, and accepted in revised form September 25, 2007.

Address correspondence to: Volker H. Haase, 700 Clinical Research Building, 415 Curie Boulevard, University of Pennsylvania, Philadelphia, Pennsylvania 19104, USA. Phone: (215) 573-1846; Fax: (215) 746-5831; E-mail: vhaase@mail.med.upenn.edu.

Debra F. Higgins and Kuniko Kimura contributed equally to this work.
1. Bohle, A., Mackensen-Haen, S., and Wehrmann, M. 1996. Significance of postglomerular capillaries in the pathogenesis of chronic renal failure. Kidney Blood Press. Res. 19:191-195.

2. Choi, Y.J., et al. 2000. Peritubular capillary loss is associated with chronic tubulointerstitial injury in human kidney: altered expression of vascular endothelial growth factor. Hum. Pathol. 31:1491-1497.

3. Fine, L.G., Bandyopadhay, D., and Norman, J.T. 2000. Is there a common mechanism for the progression of different types of renal diseases other than proteinuria? Towards the unifying theme of chronic hypoxia. Kidney Int. 57(Suppl. 75):S22-S26.

4. Orphanides, C., Fine, L.G., and Norman, J.T. 1997. Hypoxia stimulates proximal tubular cell matrix production via a TGF- beta1-independent mechanism. Kidney Int. 52:637-647.

5. Norman, J.T., Clark, I.M., and Garcia, P.L. 2000. Hypoxia promotes fibrogenesis in human renal fibroblasts. Kidney Int. 58:2351-2366.

6. Manotham, K., et al. 2004. Evidence of tubular hypoxia in the early phase in the remnant kidney model. J. Am. Soc. Nephrol. 15:1277-1288.

7. Schofield, C.J., and Ratcliffe, P.J. 2004. Oxygen sensing by HIF hydroxylases. Nat. Rev. Mol. Cell Biol. 5:343-354.

8. Sandau, K.B., Zhou, J., Kietzmann, T., and Brune, B. 2001. Regulation of the hypoxia-inducible factor 1alpha by the inflammatory mediators nitric oxide and tumor necrosis factor-alpha in contrast to desferroxamine and phenylarsine oxide. J. Biol. Chem. 276:39805-39811.

9. Zhou, J., Fandrey, J., Schumann, J., Tiegs, G., and Brune, B. 2003. NO and TNF-alpha released from activated macrophages stabilize HIF-1alpha in resting tubular LLC-PK1 cells. Am. J. Physiol. Cell Physiol. 284:C439-C446.

10. Richard, D.E., Berra, E., and Pouyssegur, J. 2000. Nonhypoxic pathway mediates the induction of hypoxia-inducible factor 1 alpha in vascular smooth muscle cells. J. Biol. Chem. 275:26765-26771.

11. Stiehl, D.P., Jelkmann, W., Wenger, R.H., and Hellwig-Burgel, T. 2002. Normoxic induction of the hypoxia-inducible factor 1 alpha by insulin and interleukin-1beta involves the phosphatidylinositol 3-kinase pathway. FEBS Lett. 512:157-162.

12. Treins, C., et al. 2002. Insulin stimulates hypoxiainducible factor 1 through a phosphatidylinositol 3-kinase/target of rapamycin-dependent signaling pathway. J. Biol. Chem. 277:27975-27981.

13. Feldser, D., et al. 1999. Reciprocal positive regulation of hypoxia-inducible factor 1alpha and insulin-like growth factor 2. Cancer Res. 59:3915-3918.

14. Bernhardt, W.M., et al. 2006. Preconditional activation of hypoxia-inducible factors ameliorates ischemic acute renal failure. J. Am. Soc. Nephrol. 17:1970-1978.

15. Eckardt, K.U., et al. 2005. Role of hypoxia in the pathogenesis of renal disease. Kidney Int. Suppl. 99:S46-S51.

16. Haase, V.H. 2006. Hypoxia-inducible factors in the kidney. Am. J. Physiol. Renal Physiol. 291:F271-F281.

17. Higgins, D.F., et al. 2004. Hypoxic induction of Ctgf is directly mediated by Hif-1. Am. J. Physiol. Renal Physiol. 287:F1223-F1232.

18. Higgins, D.F., et al. 2003. DNA oligonucleotide microarray technology identifies fisp-12 among other potential fibrogenic genes following murine unilateral ureteral obstruction (UUO): modulation during epithelial-mesenchymal transition. Kidney Int. 64:2079-2091.

19. Kietzmann, T., Roth, U., and Jungermann, K. 1999. 
Induction of the plasminogen activator inhibitor-1 gene expression by mild hypoxia via a hypoxia response element binding the hypoxia-inducible factor-1 in rat hepatocytes. Blood. 94:4177-4185.

20. Iwano, M., et al. 2002. Evidence that fibroblasts derive from epithelium during tissue fibrosis. J. Clin Invest. 110:341-350. doi:10.1172/JCI200215518.

21. Iwano, M., and Neilson, E.G. 2004. Mechanisms of tubulointerstitial fibrosis. Curr. Opin. Nephrol. Hypertens. 13:279-284.

22. Kalluri, R., and Neilson, E.G. 2003. Epithelialmesenchymal transition and its implications for fibrosis. J. Clin. Invest. 112:1776-1784. doi:10.1172/ JCI200320530.

23. Erler, J.T., et al. 2006. Lysyl oxidase is essential for hypoxia-induced metastasis. Nature. 440:1222-1226.

24. Zhong, H., et al. 1999. Overexpression of hypoxiainducible factor 1alpha in common human cancers and their metastases. Cancer Res. 59:5830-5835.

25. Krishnamachary, B., et al. 2006. Hypoxia-inducible factor-1-dependent repression of E-cadherin in von Hippel-Lindau tumor suppressor-null renal cell carcinoma mediated by TCF3, ZFHX1A, and ZFHX1B. Cancer Res. 66:2725-2731.

26. Krishnamachary, B., et al. 2003. Regulation of colon carcinoma cell invasion by hypoxia-inducible factor 1. Cancer Res. 63:1138-1143.

27. Strutz, F., et al. 1995. Identification and characterization of a fibroblast marker: FSP1. J. Cell Biol. 130:393-405.

28. Rankin, E.B., Tomaszewski, J.E., and Haase, V.H. 2006. Renal cyst development in mice with conditional inactivation of the von Hippel-Lindau tumor suppressor. Cancer Res. 66:2576-2583.

29. Ryan, H.E., et al. 2000. Hypoxia-inducible factorlalpha is a positive factor in solid tumor growth. Cancer Res. 60:4010-4015.

30. Soriano, P. 1999. Generalized lacZ expression with the ROSA26 Cre reporter strain. Nat. Genet. 21:70-71.

31. Huber, M.A., Kraut, N., and Beug, H. 2005. Molecular requirements for epithelial-mesenchymal transition during tumor progression. Curr. Opin. Cell Biol. 17:548-558.

32. Zhang, G., et al. 2007. Plasmin(ogen) promotes renal interstitial fibrosis by promoting epithelialto-mesenchymal transition: role of plasmin-activated signals. J. Am. Soc. Nephrol. 18:846-859.

33. Oda, T., et al. 2001. PAI-1 deficiency attenuates the fibrogenic response to ureteral obstruction. Kidney Int. 60:587-596.

34. Lucero, H.A., and Kagan, H.M. 2006. Lysyl oxidase: an oxidative enzyme and effector of cell function. Cell. Mol. Life Sci. 63:2304-2316.

35. Kagan, H.M., and Li, W. 2003. Lysyl oxidase: properties, specificity, and biological roles inside and outside of the cell. J. Cell. Biochem. 88:660-672.

36. Rosenberger, C., et al. 2005. Up-regulation of HIF in experimental acute renal failure: evidence for a protective transcriptional response to hypoxia. Kidney Int. 67:531-542.

37. Rosenberger, C., et al. 2002. Expression of hypoxia-inducible factor-1alpha and -2alpha in hypoxic and ischemic rat kidneys. J. Am. Soc. Nephrol. 13:1721-1732.

38. Klahr, S., and Morrissey, J. 2002. Obstructive nephropathy and renal fibrosis. Am. J. Physiol. Renal Physiol. 283:F861-F875.

39. Comerford, K.M., Wallace, T.J., Karhausen, J., Louis, N.A., Montalto, M.C., and Colgan, S.P. 2002. Hypoxia-inducible factor-1-dependent regulation of the multidrug resistance (MDR1) gene. Cancer Res. 62:3387-3394.

40. Schietke, R., et al. 2007. Hypoxic induction of lysyl oxidases leads to repression of E-cadherin - a possible link from the hypoxia-inducible factor to malignant transformation. Nephrol. Dial. Transpl. 22(Suppl. 6):233

41. Hohenstein, B., et al. 2006. Local VEGF activity but not VEGF expression is tightly regulated during diabetic nephropathy in man. Kidney Int. 69:1654-1661.

42. United States Renal Data System. 2006. Annual Data Report. http://www.usrds.org/adr.htm.

43. Bernhardt, W.M., et al. 2007. Involvement of hypoxia-inducible transcription factors in polycystic kidney disease. Am. J. Pathol. 170:830-842.

44. Ohashi, R., et al. 2002. Peritubular capillary regression during the progression of experimental obstructive nephropathy. J. Am. Soc. Nephrol. 13:1795-1805

45. Wang, A.Y., et al. 2007. Lysyl oxidase inhibition reduces rat liver fibrosis after bile duct ligation [abstract]. Gastroenterology. 132:A827.

46. Peinado, H., et al. 2005. A molecular role for lysyl oxidase-like 2 enzyme in snail regulation and tumor progression. EMBO J. 24:3446-3458.

47. Boutet, A., et al. 2006. Snail activation disrupts tissue homeostasis and induces fibrosis in the adult kidney. EMBO J. 25:5603-5613.

48. Gustafsson, M.V., et al. 2005. Hypoxia requires notch signaling to maintain the undifferentiated cell state. Dev. Cell. 9:617-628.

49. Sanchez-Elsner, T., et al. 2001. Synergistic cooperation between hypoxia and transforming growth factor-beta pathways on human vascular endothelial growth factor gene expression. J. Biol. Chem. 276:38527-38535.

50. Zhang, H., et al. 2003. Cellular response to hypoxia involves signaling via Smad proteins. Blood. 101:2253-2260

51. Esteban, M.A., et al. 2006. Regulation of E-cadherin expression by VHL and hypoxia-inducible factor. Cancer Res. 66:3567-3575.

52. Imai, T., et al. 2003. Hypoxia attenuates the expression of E-cadherin via up-regulation of SNAIL in ovarian carcinoma cells. Am. J. Pathol. 163:1437-1447.

53. Tanaka, T., et al. 2005. Cobalt promotes angiogenesis via hypoxia-inducible factor and protects tubulointerstitium in the remnant kidney model. Lab. Invest. 85:1292-1307.

54. Tanaka, T., et al. 2005. Induction of protective genes by cobalt ameliorates tubulointerstitial injury in the progressive Thy 1 nephritis. Kidney Int. 68:2714-2725.

55. Gruber, M., et al. 2007. Acute postnatal ablation of Hif-2alpha results in anemia. Proc. Natl. Acad. Sci. U. S. A. 104:2301-2306.

56. Scortegagna, M., et al. 2005. HIF-2alpha regulates murine hematopoietic development in an erythropoietin-dependent manner. Blood. 105:3133-3140.

57. Rankin, E.B., et al. 2007. Hypoxia-inducible factor-2 (HIF-2) regulates hepatic erythropoietin in vivo. J. Clin. Invest. 117:1068-1077. doi:10.1172/JCI30117.

58. Kojima, I., et al. 2007. Protective role of hypoxiainducible factor-2alpha against ischemic damage and oxidative stress in the kidney. J. Am. Soc. Nephrol. 18:1218-1226.

59. Lindenmeyer, M.T., et al. 2007. Interstitial vascular rarefaction and reduced vegf-a expression in human diabetic nephropathy. J. Am. Soc. Nephrol. 18:1765-1776.

60. Wenger, R.H., Stiehl, D.P., and Camenisch, G. 2005. Integration of oxygen signaling at the consensus HRE. Sci. STKE. 2005 :re 12

61. Ding, M., et al. 2006. Loss of the tumor suppressor Vhlh leads to upregulation of $\mathrm{Cxcr} 4$ and rapidly progressive glomerulonephritis in mice. Nat. Med. 12:1081-1087.

62. Brukamp, K., Jim, B., Moeller, M.J., and Haase, V.H. 2007. Hypoxia and podocyte-specific Vhlh deletion confer risk of glomerular disease. Am. J. Physiol. Renal Physiol. 293:F1397-1407.

63. Steenhard, B., et al. 2005. Podocyte-selective deletion of von Hippel-Lindau (VHL) protein causes albuminuria [abstract]. J. Am. Soc. Nephrol. 16:667A.

64. Rankin, E.B., et al. 2005. Inactivation of the arylhydrocarbon receptor nuclear translocator (Arnt) suppresses von Hippel-Lindau disease-associated vascular tumors in mice. Mol. Cell. Biol. 25:3163-3172.

65. Schmid, H., et al. 2006. Modular activation of nuclear factor-kappaB transcriptional programs in human diabetic nephropathy. Diabetes. 55:2993-3003.

66. MacGregor, G.R., Zambrowicz, B.P., and Soriano, P. 1995. Tissue non-specific alkaline phosphatase is expressed in both embryonic and extraembryonic lineages during mouse embryogenesis but is not required for migration of primordial germ cells. Development. 121:1487-1496.

67. Bernhardt, W.M., et al. 2006. Expression and distribution of hypoxia inducible transcription factors in mouse liver, heart and kidney [abstract]. Nephrol. Dial. Transpl. 21(Suppl. 4):17. 